This item is the archived peer-reviewed author-version of:

Hole doping and structural transformation in $C s T l_{1-x} H g_{x} C l_{3}$

\title{
Reference:
}

Retuerto Maria, Yin Zhiping, Emge Thomas J., Paria Sena Robert, Hadermann Joke, et al..- Hole doping and structural transformation in $\mathrm{CsTl}_{1-x} \mathrm{Hg}_{x} \mathrm{Cl}_{3}$

Inorganic chemistry / American Chemical Society - ISSN 0020-1669 - 54:3(2015), p. 1066-1075

Full text (Publishers DOI): http://dx.doi.org/doi:10.1021/ic502400d

To cite this reference: http://hdl.handle.net/10067/1244200151162165141 


\section{Hole doping and structural transformation in $\mathrm{CsTl}_{1-x} \mathrm{Hg}_{x} \mathrm{Cl}_{3}$ \\ Submitted in the special issue of Inorganic Chemistry in memory of John Corbett}

Maria Retuerto"\#, Zhiping Yin ${ }^{2}$, Thomas Emge ${ }^{1}$, Peter W. Stephens ${ }^{3}$, Man-Rong Li ${ }^{1}$, Tapati Sarkar ${ }^{1 \S}$, Mark $\mathrm{Croft}^{2}$, Alexander Ignatov ${ }^{2}$, Zhen Yuan ${ }^{4}$, Sijia Zhang ${ }^{4}$, Chang Qing Jin ${ }^{4}$, Robert Paria Sena ${ }^{5}$, Joke Hadermann ${ }^{5}$, Gabriel Kotliar ${ }^{2}$, Martha Greenblatt ${ }^{*}$

${ }^{1}$ Department of Chemistry and Chemical Biology, Rutgers, The State University of New Jersey, 610 Taylor Road, Piscataway, NJ 08854, USA

${ }^{2}$ Department of Physics and Astronomy, Rutgers, The State University of New Jersey, 136 Frelinghuysen Road, Piscataway, NJ 08854, USA

${ }^{3}$ Department of Physics \& Astronomy, The State University of New York, Stony Brook, NY 11794, USA

${ }^{4}$ Institute of Physics, Chinese Academy of Sciences, Number 8, Zhongguancun South Str 3, Beijing 100190, China

${ }^{5}$ EMAT, Department of Physics, University of Antwerp, Groenenborgaan, 171 2020, Antwerp, Belgium 


\begin{abstract}
$\mathrm{CsTlCl}_{3}$ and $\mathrm{CsTlF}_{3}$ perovskites have been theoretically predicted to be superconductors when properly hole doped. Both compounds have been previously prepared as pure compounds: $\mathrm{CsTlCl}_{3}$ in a tetragonal $(I 4 / m)$ and a cubic $(F m-3 m)$ polymorph, and $\mathrm{CsTlF}_{3}$ as a cubic perovskite $(F m-3 m)$. In this work, the substitution of $\mathrm{CsTlCl}_{3}$ with $\mathrm{Hg}$ is reported, in an attempt to hole dope the system and induce superconductivity. The whole series $\mathrm{CsTl}_{1-x} \mathrm{Hg}_{x} \mathrm{Cl}_{3}$ (x = 0.0, 0.1, 0.2, 0.4, 0.6 and 0.8) was prepared. $\mathrm{CsTl}_{0.9} \mathrm{Hg}_{0.1} \mathrm{Cl}_{3}$ is tetragonal as the more stable phase of $\mathrm{CsTlCl}_{3}$. However $\mathrm{CsTl}_{0.8} \mathrm{Hg}_{0.2} \mathrm{Cl}_{3}$ is already cubic with Fm-3m space group and with two different positions for $\mathrm{Tl}^{1+}$ and $\mathrm{Tl}^{3+}$. For $\mathrm{x}=0.4$ and 0.5 solid solutions could not be formed. For $\mathrm{x} \geq 0.6$ the samples are primitive cubic perovskites with one crystallographic position for $\mathrm{Tl}^{1+}, \mathrm{Tl}^{3+}$ or $\mathrm{Hg}^{2+}$. All the samples formed are insulating and there is no signature of superconductivity. X-ray absorption spectroscopy indicates that all the samples have a mixed valence state of $\mathrm{Tl}^{1+}$ and $\mathrm{Tl}^{3+}$. Raman spectroscopy shows the presence of the active Tl-Cl-Tl stretching mode over the whole series and the intensity of the $\mathrm{Tl}-\mathrm{Cl}-\mathrm{Hg}$ mode increases with increasing $\mathrm{Hg}$ content. First principle calculations confirmed that the phases are insulators in their ground state, and confirmed that $\mathrm{Hg}$ is not a good dopant in the search of superconductivity in this system.
\end{abstract}

Keywords: superconductivity, mixed valence, charge order, $\mathrm{CsTlCl}_{3}, \mathrm{CsAuCl}_{3}, \mathrm{BaBiO}_{3}$. 


\section{Introduction}

Since the discovery of superconductivity in the doped $\mathrm{BaBiO}_{3}$ compounds, many efforts have been made to find similar superconducting compounds and also to try to understand the mechanism that governs superconductivity in these types of phases. ${ }^{1,2}$ The most relevant characteristic of $\mathrm{BaBiO}_{3}$ regarding superconductivity is the charge disproportionation from $\mathrm{Bi}^{4+}$ to low valent $\mathrm{Bi}^{3+}\left(6 s^{2}\right)$, and high valent $\mathrm{Bi}^{5+}\left(6 s^{0}\right)$ cations, and charge ordering at two different positions for $\mathrm{Bi}^{3+}$ and $\mathrm{Bi}^{5+}$, resulting in some deviations from the ideal cubic perovskite structure. ${ }^{3}$ When $\mathrm{BaBiO}_{3}$ is hole doped, as in $\mathrm{Ba}_{1-x} \mathrm{~K}_{x} \mathrm{BiO}_{3}$, it becomes superconducting with a critical superconducting temperature $\left(T_{C}\right)$ of $34 \mathrm{~K}$ for $\mathrm{Ba}_{0.6} \mathrm{~K}_{0.4} \mathrm{BiO}_{3}$. ${ }^{1}$ At and below $T_{C}$, the structure transforms to the cubic perovskite phase, a symmetry that was thought to be necessary for superconductivity. ${ }^{4}$ However, recently in the similar series, $\mathrm{BaPb}_{1-x} \mathrm{Bi}_{x} \mathrm{O}_{3}$, it was observed that superconductivity was still present even in a non-cubic phase. ${ }^{5}$ The observation of superconductivity in $\mathrm{Ba}_{1-x} \mathrm{~K}_{x} \mathrm{BiO}_{3}$ or $\mathrm{BaPb}_{1-x} \mathrm{Bi}_{x} \mathrm{O}_{3}$ seems to be related to the hole doping of the $\mathrm{Bi}$ sites, which suppresses $\mathrm{Bi}^{3+} / \mathrm{Bi}^{5+}$ charge/site ordering, produces metallic behavior, and, at low temperatures, superconductivity. These observations suggest that there might be a strong relationship between the suppression of the charge ordering and superconductivity.

However, related compounds with similar charge ordering, such as $\mathrm{CsAuCl}_{3}$, do not present superconductivity, even at high pressure where structural deviations from cubic symmetry are removed and a metallic state is achieved. ${ }^{6-9}$ Recent models explained superconductivity in doped $\mathrm{BaBiO}_{3}$ by a correlation-enhanced strong electron-phonon coupling mechanism. ${ }^{2}$ Error!

Bookmark not defined. It was shown that electron-phonon coupling is weak in $\mathrm{CsAuCl}_{3}$ so that superconductivity is not expected. ${ }^{10}$ However, other calculations predicted that if the Fermi level of $\mathrm{CsAuCl}_{3}$ could be raised, then superconductivity might be achieved in the family of materials $\mathrm{ATlX}_{3}$ where $\mathrm{A}=\mathrm{Rb}$ and $\mathrm{Cs}$ and $\mathrm{X}=\mathrm{Cl}, \mathrm{F}, \mathrm{Br}$; when the compounds are hole doped. ${ }^{10,11}$ For example, $\mathrm{CsTlF}_{3}$ and $\mathrm{CsTlCl}_{3}$ were theoretically predicted to be superconducting upon the optimal $\sim 0.35 /$ f.u. hole doping and moderate pressures of $\sim 10$ and $\sim 2 \mathrm{GPa}$, with predictions of $T_{C}$ at $\sim 30$ and $\sim 20 \mathrm{~K}$, respectively. ${ }^{10}$ 
Recently we were able to synthesize these two materials, $\mathrm{CsTlF}_{3}$ and $\mathrm{CsTlCl}_{3}$ in the perovskite structure predicted to be thermodynamically stable. ${ }^{12} \mathrm{CsTlF}_{3}$ was obtained as a white polycrystalline material with cubic perovskite structure and $\mathrm{Tl}^{1+}$ and $\mathrm{Tl}^{3+}$ in separate crystallographic positions. $\mathrm{CsTlCl}_{3}$ was obtained as orange single crystals with two different morphologies: A) a tetragonal phase, with separate positions for $\mathrm{Tl}^{1+}$ and $\mathrm{Tl}^{3+}$ in alternate octahedra of different sizes; and B) a cubic phase, also with different sites for $\mathrm{Tl}^{1+}$ versus $\mathrm{Tl}^{3+}$. In both polymorphs there is some site disorder at the $\mathrm{Tl}^{1+}$ position. Thus, $\mathrm{CsTlF}_{3}$ and two phases of $\mathrm{CsTlCl}_{3}$ present $\mathrm{Tl}^{1+} / \mathrm{Tl}^{3+}$ charge ordering, which is a potentially good starting point for superconductivity. In addition, electronic band structure calculations showed that the optical band gap of $\mathrm{CsTlCl}_{3}$ has the required energy if charge ordering is present, and that hole doping of this material could lead to superconductivity. ${ }^{12}$

However, all of our efforts to hole dope $\mathrm{CsTlCl}_{3}$ by topotactically forming $\mathrm{Cs}_{1-x} \mathrm{TlCl}_{3}$ or by electron doping chemically, e.g., $\mathrm{CsTlCl}_{3-x}(\mathrm{O}, \mathrm{S}, \mathrm{N})_{x}$, to achieve superconductivity have not been successful so far. Therefore, analogous to the formation of $\mathrm{BaBi}_{1-\mathrm{x}} \mathrm{Pb}_{\mathrm{x}} \mathrm{O}_{3},{ }^{13}$ we attempted hole doping by substitution of $\mathrm{Hg}^{2+}\left(6 s^{0}\right)$ for the $\mathrm{Tl}^{1+} / \mathrm{Tl}^{3+}$ positions and prepared the entire $\mathrm{CsTl}_{1-}$ ${ }_{x} \mathrm{Hg}_{x} \mathrm{Cl}_{3}(0.0 \leq x \leq 1.0)$ series. This was a significant achievement, since this class of chlorides are difficult to dope and, any partial doping of $\mathrm{CsAuCl}_{3}$ has not yet been successful.

\section{Experimental Section}

$\mathrm{CsTl}_{1-x} \mathrm{Hg}_{x} \mathrm{Cl}_{3}(0.0 \leq x \leq 1.0)$ series were prepared by mixing stoichiometric quantities of $\mathrm{CsCl}$, $\mathrm{TlCl}, \mathrm{TlCl}_{3} \cdot x \mathrm{H}_{2} \mathrm{O}$ and $\mathrm{HgCl}_{2}$ inside a glove box in order to avoid the decomposition of $\mathrm{TlCl}_{3} \cdot x \mathrm{H}_{2} \mathrm{O}$ into $\mathrm{TlCl}$, due to the hygroscopic character of the starting materials (e.g., the solid $\mathrm{TlCl}_{3} \cdot x \mathrm{H}_{2} \mathrm{O}$ reagent was deliquescent upon exposure to moist air) and also due to the high toxicity of thallium. The starting materials were mixed, ground, placed in a silica tube and sealed under vacuum; then they were heated to $600{ }^{\circ} \mathrm{C}$ for 12 hours and finally the temperature was decreased at a rate of $5{ }^{\circ} \mathrm{C} / \mathrm{min}$, to ambient temperature. The final products were orange- intergrown single crystals of $\sim 1-2 \mathrm{~mm}$. Powder x-ray diffraction (PXRD) patterns of the obtained compounds are shown in Fig. 1, and single crystals of $\mathrm{CsTl}_{0.4} \mathrm{Hg}_{0.6} \mathrm{Cl}_{3}$ are illustrated in the upper 
inset of Fig. 1. The PXRD characterization was carried out on a Bruker D8 X-ray diffractometer $(\mathrm{Cu} \mathrm{K}, \lambda=1.5418 \AA$ Á). Synchrotron powder x-ray diffraction (SXRD) data for the Rietveld refinement of the series were collected at room temperature at the National Synchrotron Light Source (NSLS) at Brookhaven National Laboratory using the X16C beam line and a wavelength of 0.7001 Á. Rietveld refinements were carried out with TOPAS software. ${ }^{14}$ Powder samples were diluted with approximately $90 \%$ by weight diamond powder, to control absorption in the $1 \mathrm{~mm}$ (nominal) Lindemann capillaries, sealed in an inert atmosphere. Single-crystal x-ray data (SCD) were collected on a Bruker Smart APEX CCD diffractometer with graphite monochromatized Mo $\mathrm{K}_{\alpha}$ radiation $(\lambda=0.71073 \AA)$ at $100 \mathrm{~K}$. The data were corrected for Lorenz effects and polarization, and absorption, the latter by a multi-scan (SADABS) ${ }^{15}$ method. The structure was solved by Patterson methods (SHELXS86). ${ }^{16}$ All atoms were refined by use of SHELXL (2013) and JANA2006 programs. ${ }^{17}$

X-ray absorption spectra (XAS) measurements were made in both fluorescence and transmission modes on beamline X-19A at the NSLS with a Si(111) double-crystal monochromator. ${ }^{18,19}$ The $\mathrm{Tl}-\mathrm{L}_{3}$ measurements were made in both transmission and fluorescence modes with simultaneousrun of $\mathrm{Tl}_{2} \mathrm{O}_{3}$ standard for energy calibration. The low energy of the $\mathrm{Cl}-\mathrm{K}$ edge measurements were possible due to the $100 \mu \mathrm{m}$ Be beamline window and a specially designed $\mathrm{He}$ atmosphere fluorescence mode chamber. Energy calibration in the $\mathrm{Cl}-\mathrm{K}$ measurements was made by frequent running of the same standard in the sequence of scans. The data were processed with the standard linear pre- and post-edge background subtraction. The absorption coefficient $(\mu)$ was normalized to 1.0 over an average energy region well above the edge. The Raman spectra of this $\mathrm{CsTl}_{1-x} \mathrm{Hg}_{x} \mathrm{Cl}_{3}$ series were recorded with the Renishaw Micro-Raman Spectroscopy System with a laser wavelength of $532 \mathrm{~nm}$ and an output power of $100 \mathrm{~mW}$.

\section{Results and Discussion}

Elemental analyses were carried out by inductively coupled plasma (ICP) mass spectrometry to determine the ratio of $\mathrm{Tl}$ and $\mathrm{Hg}$ in each sample. The composition determined for several crystals was: $80(1) \% \mathrm{Tl} / 20(1) \% \mathrm{Hg}$ for nominal $\mathrm{CsTl}_{0.8} \mathrm{Hg}_{0.2} \mathrm{Cl}_{3} ; 61(1) \% \mathrm{Tl} / 39(1) \% \mathrm{Hg}$ for nominal 
$\mathrm{CsTl}_{0.6} \mathrm{Hg}_{0.4} \mathrm{Cl}_{3} ; 46(1) \% \mathrm{Tl} / 54(1) \% \mathrm{Hg}$ for nominal $\mathrm{CsTl}_{0.4} \mathrm{Hg}_{0.26} \mathrm{Cl}_{3}$; and 23(1)\% $\mathrm{Tl} / 77(1) \% \mathrm{Hg}$ for nominal $\mathrm{CsTl}_{0.2} \mathrm{Hg}_{0.8} \mathrm{Cl}_{3}$. In all cases Cs atoms are stoichiometric within the error range of the respective formula given above. Due to the close agreement of the chemically analysed compositions with the nominal compositions, the nominal compositions are employed to designate the compositions of the samples. The X-ray fluorescence (XRF) analyses of the above samples were consistent with the respective ICP results.

\section{Crystal Structure}

Fig. 1 shows the PXRD patterns of the $\mathrm{CsTl}_{1-x} \mathrm{Hg}_{x} \mathrm{Cl}_{3}(x=0.0 .0 .1,0.2,0.4,0.6,0.8$ and 1.0) series. For pure $\mathrm{CsTlCl}_{3}$, we have previously reported two phases: a body-centered tetragonal phase $(\mathrm{I} 4 / \mathrm{m})$ and a relatively less stable $(\mathrm{F} m-3 \mathrm{~m})$ cubic phase. ${ }^{12}$ A similar tetragonal structure was observed for the $x=0.1$ compound. The PXRD pattern of the tetragonal phase contains the "split peaks" features consistent with the tetragonal phase (I4/m) found for $x=0.0$, namely $a$ ' $\approx$ $b^{\prime} \approx 17 \AA$ and $c^{\prime} \approx 11 \AA$, for $a^{\prime}=5^{1 / 2} a$ and $c^{\prime}=c$ superlattice, ${ }^{12}$ with respect to the known $a \approx$ $b \approx 7 \AA, c \approx 11 \AA$ of the tetragonal $(\mathrm{I} / 4 / \mathrm{mmm}) \mathrm{CsMCl}_{3}(\mathrm{M}=\mathrm{Au}, \mathrm{Ag})$ phases. ${ }^{20,21}$ The formation of this superlattice is expected due to the lone pair effect of the $\mathrm{Tl}^{1+}\left(6 \mathrm{~s}^{2}\right)$ cation in this tetragonal site, which allows for a distortion in the structure and the tilting of the $\mathrm{TlO}_{6}$ octahedral with respect to an undistorted cubic sublattice. For $x \geqslant 0.2$, the PXRD patterns (Fig. 1) indicate that the structures have cubic symmetry. Thus, the substitution of small amounts of $\mathrm{Hg}$ for $\mathrm{Tl}$ in $\mathrm{CsTl}_{1-x} \mathrm{Hg}_{x} \mathrm{Cl}_{3}$ is apparently sufficient to stabilize a cubic perovskite instead of a lower-symmetry tetragonal phase.

The lower inset of Fig. 1 shows the $10^{\circ}<2 \theta<20^{\circ}$ region of the PXRD patterns. Comparing the PXRD data of the cubic samples $(x=0.2,0.4,0.6 .0 .8$ and 1.0) it was observed that an Fcentered cubic reflection exists at $2 \theta=14^{\circ}$ only for $x \leqslant 0.4$. This observed peak and the less intense peaks expected at $2 \theta=27^{\circ}$ and $36^{\circ}$, are the result of inequivalent $\mathrm{B}$ sites for $\mathrm{Tl}$ in this perovskite at $\left(\begin{array}{lll}0 & 0 & 0\end{array}\right)$ and $\left(1 / 22^{1 / 2}{ }^{1 / 2}\right)$, as found for the $\mathrm{Fm}-3 m$ phase of $\mathrm{Cs}_{2} \mathrm{Au}^{+1} \mathrm{Au}^{+3} \mathrm{Cl}_{6}$, namely, $\mathrm{Au}^{+3}$ at $\left(\begin{array}{lll}0 & 0 & 0\end{array}\right)$ and $\mathrm{Au}^{+1}$ at $\left(1 / 2{ }^{1 / 2} 1 / 2\right) .{ }^{22}$ We have previously observed the same F-centering in the cubic phase of $\mathrm{CsTlCl}_{3}$ where weak peaks at $14^{\circ}$ and $27^{\circ}$, are explained by the presence of $\mathrm{Tl}^{1+}$ 
and $\mathrm{Tl}^{3+}$ in two different crystallographic sites. This should be expected since $\mathrm{Tl}^{1+}$ and $\mathrm{Tl}^{3+}$ have very different ionic sizes $\left(1.5 \AA\right.$ and $0.885 \AA$, respectively). ${ }^{23}$ Also, the lone pair effect of $\mathrm{Tl}^{1+}$ is expected to create a very different environment for its $\mathrm{Tl}^{1+} \mathrm{Cl}_{6}$ octahedra compared to $\mathrm{Tl}^{3+} \mathrm{Cl}_{6}$.

For $x \geqslant 0.6$ the lattice is primitive in a cubic perovskite model $\left(\mathrm{CaTiO}_{3}\right.$ structural type, $\left.\mathrm{P} m-3 m\right)$ with one $\mathrm{B}$ site for all $\mathrm{Tl}$ or $\mathrm{Hg}$ atoms. This finding could be of great significance, because if the two different positions for $\mathrm{Tl}^{1+}$ and $\mathrm{Tl}^{3+}$ no longer exist; then we may have been able to dope the cubic $\mathrm{CsTlCl}_{3}$ phase and prepare for the first time a compound with single-valent $\mathrm{Tl}^{2+}$ at one position. This would be a good starting point in the search for superconductivity in this type of material, in the manner predicted by Z. P Yin et al. ${ }^{10}$ and experimentally observed in K-doped $\mathrm{BaBiO}_{3} .{ }^{1}$ However, it is more likely that both $\mathrm{Tl}^{1+}$ and $\mathrm{Tl}^{3+}$ occupy the single $\mathrm{B}$ position, or that an overabundance of $\mathrm{Hg}^{2+}$ in one site masks the true positions of $\mathrm{Tl}^{1+}$ and $\mathrm{Tl}^{3+}$ wherever they are. The results of the Raman spectroscopy helped us confirm the presence of $\mathrm{Tl}^{1+}$ and $\mathrm{Tl}^{3+}$ rather than the intermediate $\mathrm{Tl}^{2+}$ (see below).

We were able to use single crystal diffraction (SCD) results to find precise models of the primitive and F-centered phases and to determine the extent of atomic site disorder. We collected data on different crystals of the same batches of $x=0.2,0.6$ and 0.8 for $\mathrm{CsTl}_{1-x} \mathrm{Hg}_{x} \mathrm{Cl}_{3}$. We do not report SCD results here for the $x=0.4$ material, because the SXRD strongly indicated that it was most likely not a single phase (see below).

Consistent with the PXRD result, the SCD data of $\mathrm{CsTl}_{0.8} \mathrm{Hg}_{0.2} \mathrm{Cl}_{3}(x=0.2)$ agrees best with the F $m-3 m$ double perovskite model, very similar to the one we previously reported for cubic $\mathrm{CsTlCl}_{3}$ and to that reported for $\mathrm{CsAuCl}_{3}$. In our model for the $x=0.2$ phase, the Cs site at $8 c(1 / 4$ $1 / 4^{1 / 4}$ ) has a slight disorder, as evidenced by small, but significant (about $3 \mathrm{e}-/ \AA^{3}$ ), residuals in the difference electron density map, but refinement of any off-site atom failed due to large correlation coefficients, as expected from the small distance (about $0.5 \AA$ ) to the $8 c$ site. The $\mathrm{Tl}$ site at $4 a\left(\begin{array}{lll}0 & 0 & 0\end{array}\right)$ was ordered and fully occupied and was assigned as $\mathrm{Tl}$ only. The $\mathrm{Hg}(2 \mathrm{~A}) / \mathrm{Tl}(2 \mathrm{~B})$ site at $4 b(1 / 200)$ was noticeably split, with the majority of the electron density at $24 e$ position ( $\mathrm{x} 00$ ) with $\mathrm{x}=0.5721(10)$, and much less electron density at the $4 b$ position $(1 / 20$ 
0 ). This overall site was modelled with $\mathrm{Hg}$ at the $4 b$ site and $\mathrm{Tl}$ at the $24 e$ site and initially refined to 14 and $86 \%$ occupancy, respectively. For the final cycles of refinement, the $4 b$ and $24 e$ sites were given different isotropic displacement parameters but fixed at 1/6 and 5/6 occupancy, respectively, in order to: A) agree with the $\mathrm{Cl}$ atom site disorder of 5:1 (see below); B) be consistent with 1 out of $6 \mathrm{Tl}-\mathrm{Cl}$ bonds being unrealistic $(<2 \AA)$ and ignored here, resulting in 4- or 5-coordinate $\mathrm{Tl}$; and $\mathrm{C}$ ) yield the overall stoichiometry $\mathrm{CsTl}_{0.8} \mathrm{Hg}_{0.2} \mathrm{Cl}_{3}(x=0.2)$. The correlation matrix element for $\mathrm{U}^{11} \mathrm{Hg}(2 \mathrm{~A}) / \mathrm{U}^{11} \mathrm{Tl}(2 \mathrm{~B})$ was 0.516 (program SHELXL v. 2014_3).The $\mathrm{Cl}(1 \mathrm{~A})$ site at $24 e$ ( $\mathrm{x} 0$ 0) with $\mathrm{x}=0.2413$ (9) was ordered, but not fully occupied, and a second $\mathrm{Cl}(1 \mathrm{~B})$ site was found at $48 h$ ( $\mathrm{x}$ x 0 ) with $\mathrm{x}=0.165(4)$, at a substantial distance (2 $\AA$ A) away. After restrained refinement, it was found that the occupancy of $\mathrm{Cl}(1 \mathrm{~A})$ and $\mathrm{Cl}(1 \mathrm{~B})$ were approximately 5/6 and 1/6 and were subsequently set equal to these values, respectively, and the same isotropic displacement parameter was refined for $\mathrm{Cl}(1 \mathrm{~A})$ and $\mathrm{Cl}(1 \mathrm{~B})$. The final atomic coordinates and anisotropic displacement parameters are listed in Table 1 and the bond distances and angles are in Table 2. The $\mathrm{Tl}(1)-\mathrm{Cl}(1 \mathrm{~A})$ distance of 2.608(10) $\AA$ is significantly shorter than the $\mathrm{Hg}(2 \mathrm{~A})-\mathrm{Cl}(1 \mathrm{~A})$ of $2.795(10) \AA$, consistent with $\mathrm{Tl}^{3+}$ at the $\mathrm{Tl}(1)$ site and $\mathrm{Hg}^{2+}$ at the $\mathrm{Hg}(2 \mathrm{~A})$ site, although $\mathrm{Hg}$ cannot be distinguished from $\mathrm{Tl}$ by this $\mathrm{X}$-ray experiment and are only assigned here. Both distances are smaller than expected by Shannon, ${ }^{23}$ as happens in other similar phases, such as $\mathrm{CsAuCl}_{3}$ or $\mathrm{ClAgCl}_{3}{ }^{21,22} \mathrm{Also}$, it has been found that the off-site residual density was more appropriately modelled by site disorder (here, in program SHELXL, which yields ranges of bond geometries as in Table 2) rather than by use of anharmonic displacement parameters, such as those available in refinement program JANA2006 (which yields average bond geometries).

Rietveld fits of the SXRD results, shown in Fig. 2, support this interpretation, but show that all of the powder samples have two coexisting phases. The $x=0.1$ sample is a 50:50 mixture of the tetragonal phase with $a=17.1498(3) \AA, c=11.0823(3) \AA$ and a cubic $(F m-3 m)$ phase with $a=$ $10.9042(3) \AA$. The $x=0.2$ sample is $90 \% F m-3 m$ phase with $a=10.9019(3) \AA$, and $10 \%$ with $a$ $=10.8206(3) \AA$. The $x=0.4$ sample (not shown) is a roughly 50-50 mixture of $(F m-3 m)$ phases with $a=10.9026(3) \AA$ and 10.8616(3) A; interestingly, the odd-order peaks are much stronger in the phase with larger lattice parameter, which is essentially identical to the lattice parameter of 
the majority phase of the $x=0.2$ sample. The split $4 b$ site model described above is required to explain the intensity of the odd-order peaks; differences in valence or occupancy of the two Tl sites alone cannot account for the measured SXRD patterns.

The SCD data of different crystals of $x=0.6$ and $0.8\left(\mathrm{CsTl}_{1-x} \mathrm{Hg}_{x} \mathrm{Cl}_{3}\right)$ have the well-known primitive cubic perovskite model, $\mathrm{P} m-3 m$, with $\mathrm{Tl}$ or $\mathrm{Hg}$ atoms located at or near the $1 b(1 / 21 / 21 / 2)$ site, and Cs atoms at $1 a\left(\begin{array}{lll}0 & 0 & 0\end{array}\right)$ sites. The $\mathrm{Tl} / \mathrm{Hg}(1 \mathrm{~A})$ site at $1 b\left(1 / 2 \frac{1}{2} \frac{1 / 2}{2}\right)$ was disordered, with partial occupancy at $1 b$ and a sextet of positions about the $1 b$ site modelled by $\mathrm{Tl} / \mathrm{Hg}(1 \mathrm{~B})$ at $6 f(\mathrm{x}$ $1 / 21 / 2$ ) with $x=0.35$ and occupancies of 0.02 or less for both $\mathrm{CsTl}_{0.4} \mathrm{Hg}_{0.6} \mathrm{Cl}_{3}$ and $\mathrm{CsTl}_{0.2} \mathrm{Hg}_{0.8} \mathrm{Cl}_{3}$. In the case of $\mathrm{CsTl}_{0.4} \mathrm{Hg}_{0.6} \mathrm{Cl}_{3}$ the occupancy at $1 b$ was refined to a slightly larger value than that of $\mathrm{CsTl}_{0.2} \mathrm{Hg}_{0.8} \mathrm{Cl}_{3}$ (see Table 2). The $\mathrm{Cl}$ site in these two $\mathrm{Pm}-3 m$ structures appears to be ordered and fully occupied at a single site $3 c\left(0 \frac{1}{2} \frac{1}{2}\right)$. The $\mathrm{Tl} / \mathrm{Hg}-\mathrm{Cl}$ distance for either $\mathrm{P} m-3 m$ structure was about $2.69 \AA$ and in between those for the Fm-3m structure. The coordination geometries of $\mathrm{x}=0.6$ and $\mathrm{x}=0.8$ are very similar to each other and do not appear to correlate with $\mathrm{Hg}$ : $\mathrm{Tl}$ ratio in any way. The final atomic coordinates and anisotropic displacement parameters are listed in Table 1 and the main $\mathrm{Tl} / \mathrm{Hg}(1 \mathrm{~A})$ and minor $\mathrm{T} 1 / \mathrm{Hg}(1 \mathrm{~B})$ site bond distances and angles in Table 2. As shown in Table 1, the cell parameters decrease as the content of $\mathrm{Hg}$ was increased, correlating well with the values of the ionic radii, $\mathrm{Tl}^{1+}\left(r^{\mathrm{VI}}: 1.5 \AA\right), \mathrm{Tl}^{3+}\left(r^{\mathrm{VI}}: 0.885 \AA\right)$, for an average of 1.19 $\AA$ for $\mathrm{Tl}$, and $\mathrm{Hg}^{2+}\left(r^{\mathrm{VI}}: 1.02 \AA\right){ }^{23}$

Attempts to prepare $\mathrm{CsTl}_{0.5} \mathrm{Hg}_{0.5} \mathrm{Cl}_{3}$ were not successful, which indicates that it may not be possible to form the full range of the solid solutions in $\mathrm{CsTl}_{1-x} \mathrm{Hg}_{x} \mathrm{Cl}_{3}$. Also, these samples were prepared as single crystals from self-flux, and for $\mathrm{CsTl}_{0.6} \mathrm{Hg}_{0.4} \mathrm{Cl}_{3}$ different compositions are formed at different locations inside the quartz tube, e.g., the crystals grown on the walls of the tube have cell data similar to $\mathrm{CsTl}_{0.4} \mathrm{Hg}_{0.6} \mathrm{Cl}_{3}$, while the ones grown in the middle of the flux have cell data similar to $\mathrm{CsTl}_{0.8} \mathrm{Hg}_{0.2} \mathrm{Cl}_{3}$.

Fig. 2c shows the SXRD data of $x=0.6\left(\mathrm{CsTl}_{0.4} \mathrm{Hg}_{0.6} \mathrm{Cl}_{3}\right)$. Contrary to the PXRD and the SCD results, the synchrotron data shows very small F-centering peaks at $6.38^{\circ}, 16.13^{\circ}$, and $19.25^{\circ}$. These are too weak to support the split-site model, but could arise from valence or vacancy 
ordering on the two Tl sites. The discrepancy could arise because the harvest region of this sample was close to the area where the solid solution was not formed and multiple phases may form. Doubled cell parameters of 10.856(5) $\AA$ and 10.884(5) $\AA$ were observed in that powder. To confirm that the $\mathrm{Pm}-3 m$ crystals contain $\mathrm{Tl}$ and $\mathrm{Hg}$, and are not just pure $\mathrm{CsHgCl}_{3}$; we carried out XRF and we observed $\mathrm{Tl} 36.2(2): \mathrm{Hg}$ 63.8(2) \%. The presence of $\mathrm{Tl}$ was also indicated by the orange color, compared to the white/colorless crystals of pure $\mathrm{CsHgCl}_{3}$.

Crystals of the compound with $x=0.8\left(\mathrm{CsTl}_{0.2} \mathrm{Hg}_{0.8} \mathrm{Cl}_{3}\right)$ were orange in color but visibly lighter color crystals, as may be expected due to the relative reduction of Tl color centers. The SXRD of this compound refined well in the simple perovskite model Pm-3m, as we previously observed by SCD, and we used that model for the refinement, which gave a refined cell parameter of 5.4161(1) ̊.

We measured the magnetic properties of all the members of the series between 5-300 K, but none were magnetically interesting and the signals are so low that we cannot trust the measurements and separate them from the background, or the signal of the plastic sample holder; thus the samples are not magnetic.

\section{$X$-ray Absorption Spectroscopy $(X A S)$}

We performed XAS to observe if there is change in Tl oxidation state as the content of mercury increases, and also to see if there is any change in the shape of the curves indicating an evolution from mixed $\mathrm{Tl}^{1+} / \mathrm{Tl}^{3+}$ state to a single $\mathrm{Tl}^{2+}$ state. Thallium usually presents $\mathrm{Tl}^{1+}$ and $\mathrm{Tl}^{3+}$ valence states: $\mathrm{Tl}^{3+}$ involves two $6 s$-orbital holes and $\mathrm{Tl}^{1+}\left(6 s^{2}\right)$ none. The $\mathrm{Tl}-\mathrm{L}_{3}$ edge XAS signature of $\mathrm{Tl}^{1+}$ to $\mathrm{Tl}^{3+}$ change is a chemical shift of the main edge to higher energy and the appearance of a shoulder pre-edge feature due to transitions into the empty $6 s$ hole states. ${ }^{12}$ These same signatures are observed in the $\mathrm{Bi}-\mathrm{L}_{3}$ edge from $\mathrm{Bi}^{3+}$ to $\mathrm{Bi}^{5+} \cdot{ }^{24-26}$ In Fig. 3a the Tl- $\mathrm{L}_{3}$ edge spectra for the perovskite compounds $\mathrm{CsTlCl}_{3}$ (-c, cubic and $-\mathrm{t}$ tetragonal) and $\mathrm{CsTl}_{0.2} \mathrm{Hg}_{0.8} \mathrm{Cl}_{3}$ are compared to those of $\mathrm{Tl}^{1+}$ and $\mathrm{Tl}^{3+}$ standards with the pre-edge energy region being noted. The energy where the absorption coefficient first rises to the $\mu \sim 0.5$ value can be used to compare the 
nominal chemical shift of such spectra. The chemical shift of the $\mathrm{CsTlCl}_{3}$ structures are clearly intermediate between those of the $\mathrm{Tl}^{1+}$ and $\mathrm{Tl}^{3+}$ standards supporting its intermediate valence. ${ }^{12}$ Also the cubic and tetragonal forms have nearly identical spectra and will therefore not be discussed separately hereafter in the XAS discussions. The chemical shift of $\mathrm{CsTl}_{0.2} \mathrm{Hg}_{0.8} \mathrm{Cl}_{3}$ appears displaced somewhat to higher energy but still falls in the intermediate valence range. The $\mathrm{Tl}-\mathrm{L}_{3}$ edges of various other $x$-compositions in the $\mathrm{CsTl}_{1-x} \mathrm{Hg}_{x} \mathrm{Cl}_{3}$ series (not shown) have chemical shifts falling (with scatter) within these two extremes without manifesting a definitive systematic trend. Thus the retention of valence mixing in this series was indicated. Focusing on the pre-edge region of the spectra in Fig. 3a one notes pronounced pre-edge shoulder features consistent with the presence of two 6s-orbital holes $\left(6 \mathrm{~s}^{0}\right.$ configuration) in the $\mathrm{Tl}^{3+}$ compounds, $\mathrm{Tl}_{2} \mathrm{O}_{3}$ and $\mathrm{Cs}_{2} \mathrm{TlCl}_{5}$. In contrast the $\mathrm{Tl}^{1+} \mathrm{Cl}$ spectrum exhibits a monotonic concave upward curvature over the entire pre edge region, consistent with the absence of any 6 s hole states $\left(6 s^{2}\right.$ configuration).

The Cl-K near edge is dominated by dipole allowed $1 s$-to- $2 p$ transitions. The Cl-K near edges for a series of $\mathrm{Cl}$ compounds is shown in Fig. 3b. The simple structureless main-edge rise and broad peak for $\mathrm{Cl}^{1-}$ in $\mathrm{TlCl}$ is typical for the ionic bonding and filled $2 p$ orbitals in these materials. The $\mathrm{Cl}^{5+}$ in the $\mathrm{KClO}_{3}$ spectrum, on the other hand, displays an intense "white line" feature due to transitions into the now empty $2 p$ orbitals, along with a very substantial chemical shift of the main edge to higher energy. The most striking structure in the other spectra in Fig. $3 \mathrm{~b}$ is the extremely intense pre-edge features shifted well below the main edge rise. In transition metal compounds such $\mathrm{Cl}-\mathrm{K}$ pre-edge features, associated with the metal $d$-orbital states hybridized with the ligand Cl-p states are common. Indeed the d-orbital sub-splitting, bandwidths, and energies have been studied with $\mathrm{Cl}-\mathrm{K}$ XAS. ${ }^{27,28}$ In the $\mathrm{Tl}$ compounds considered here, such preedge features are associated with transitions into Cl- $2 p$ states hybridized with Tl- $6 s$ hole states. ${ }^{12}$ Note that in the filled- $6 s^{2} \mathrm{TlCl}$ spectrum no hint of the pre-edge feature was present. In the $\mathrm{Cl}-\mathrm{K}$ edges of the $\mathrm{CsHgCl}_{3}$ perovskite and the $\mathrm{HgCl}_{2}$ standard, the prominent $6 s$-hole pre edge feature was shifted less far below the main edge because of the smaller binding energy of the $\mathrm{Hg}-6 \mathrm{~s}$ states relative to those of the Tl-6s which see a larger effective core charge. The pre-edge of $\mathrm{Tl}^{3+}$ in $\mathrm{Cs}_{2} \mathrm{TlCl}_{5}$ in Fig. $3 \mathrm{~b}$ manifests the pre-edge feature with the highest spectral intensity and the 
simplest structure. The $\mathrm{CsTlCl}_{3}$-t and -c pre-edge features are reproducibly, albeit slightly, shifted to lower energy and exhibit a slightly lower spectral intensity in the pre-edge peak. Both spectra however evidence excess intensity and additional substructure between the peak and the main edge rise. See for examples the less deep minimums in intensity in the pre-edge region and the bulge most prominently observed on the rising edge of $\mathrm{CsTlCl}_{3}-\mathrm{t}$ spectrum. Although detailed electronic structure calculations will be required to clarify these spectral features there is a definite disparity from pure $\mathrm{Tl}^{3+}$ standard behaviour. In Fig. $3 \mathrm{c}$ the evolution of the pre-edge feature region as a function of $\mathrm{Hg}$ substitution in the $\mathrm{CsTl}_{1-x} \mathrm{Hg}_{x} \mathrm{Cl}_{3}$ series is shown. The systematic transfer of intensity from the Tl-6s, A-feature to the $\mathrm{Hg}-6 s$, B feature with increasing $x$ is dramatically apparent. Indeed this appears to be nearly a text-book example of the spectral evolution expected with the $x$-variation in the fraction of the A vs. B transition channels.

\section{Raman Spectroscopy}

Fig. 4 shows the Raman data of $\mathrm{CsTl}_{1-x} \mathrm{Hg}_{x} \mathrm{Cl}_{3}$. The $\mathrm{Tl}-\mathrm{Cl}-\mathrm{Tl}$ and $\mathrm{Hg}-\mathrm{Cl}-\mathrm{Hg}$ bond phonon frequencies are expected to be within a few $\mathrm{cm}^{-1}$ of each other and situated around $270 \mathrm{~cm}^{-1}$. In the case of monoclinic $\mathrm{CsHgCl}_{3}$ this frequency was known to be the Cl-breathing mode for $\mathrm{Hg}$ $\mathrm{Cl}-\mathrm{Hg} .{ }^{29}$ When $\mathrm{CsHgCl}_{3}$ is in a cubic phase, this Cl-breathing mode is not Raman-active due to symmetry, but can be active if we have the non-symmetrical and active $\mathrm{Tl}^{1+}-\mathrm{Cl}-\mathrm{Tl}^{3+}$ mode present, rather than the symmetrical $\mathrm{Tl}^{2+}-\mathrm{Cl}_{-} \mathrm{Tl}^{2+}$ mode. Since these frequencies are indeed observed in all the members of the $\mathrm{CsTl}_{1-x} \mathrm{Hg}_{x} \mathrm{Cl}_{3}$ series, we most likely have the $\mathrm{Tl}^{1+}-\mathrm{Cl}_{-} \mathrm{Tl}^{3+}$ modes active here.

Another peak appeared around $240 \mathrm{~cm}^{-1}$ which should be related to the $\mathrm{Hg}-\mathrm{Cl}-\mathrm{Tl}$ bond with a calculated phonon frequency of $230 \mathrm{~cm}^{-1}$. This frequency can be Raman active for any crystal symmetry, corresponds to $\mathrm{Hg}-\mathrm{Cl}-\mathrm{Tl}^{3+}$ or $\mathrm{Hg}-\mathrm{Cl}-\mathrm{Tl}^{1+}$, and can be computed by assuming a Fm-3m structure with $\mathrm{Tl}$ at $\left(\begin{array}{lll}0 & 0 & 0\end{array}\right)$ and $\mathrm{Hg}$ at $\left(\begin{array}{llll}1 / 2 & 1 / 2 & 1 / 2\end{array}\right)$. This peak increased as the content of $\mathrm{Hg}$ increased and was very noticeable for $\mathrm{CsTl}_{0.2} \mathrm{Hg}_{0.8} \mathrm{Cl}_{3}$, where there are more $\mathrm{Tl}-\mathrm{Cl}-\mathrm{Hg}$ interactions than Tl-Cl-Tl. . 


\section{First-Principle Calculations}

To gain further insights into how the $\mathrm{Hg}$ doping changes the crystal structure and electronic structure of this class of materials, we carried out first-principles density functional theory calculations with both the generalized gradient approximation (the PBE version) ${ }^{30}$ and the screened hybrid (the HSE06 version) ${ }^{31}$ exchange-correlation functional as implemented in the VASP code. ${ }^{32}$ First of all, we show in Fig. 5 the band structures of the parent compound $\mathrm{CsTlCl}_{3}$ and $\mathrm{CsHgCl}_{3}$. As reported in Ref.12, the face-centered cubic parent compound $\mathrm{CsTlCl}_{3}$ is an insulator with an indirect band gap of $1.2 \mathrm{eV}$ and an optical gap of $1.9 \mathrm{eV}$ within the HSE06 approach (see Fig.5a). The band gap is between the occupied $\mathrm{Tl}^{1+} 6 s$ band and the empty $\mathrm{Tl}^{3+} 6 s$ band. Using the experimental simple cubic perovskite structure with lattice constant $a=5.41 \AA$ for $\mathrm{CsHgCl}_{3}$, DFT-HSE06 calculations predict a $0.6 \mathrm{eV}$ indirect band gap and a $1.8 \mathrm{eV}$ optical gap, which is between the occupied $\mathrm{Cl} 3 p$ bands and the empty $\mathrm{Hg} 6 s$ band (see Fig.5b). This prediction is consistent with the experimental observation of insulating behaviour of $\mathrm{CsHgCl}_{3}$. In contrast, DFT-PBE calculations predicts that $\mathrm{CsHgCl}_{3}$ is metallic (but is on the border of a metal-insulator transition), which is inconsistent with experiment. We note however that the simple cubic perovskite structure of $\mathrm{CsHgCl}_{3}$ is unstable against the Jahn-Teller distortion of $\mathrm{HgCl}_{6}$ octahedra in both DFT-PBE and DFT-HSE06 approaches, which predict that the planar and apical $\mathrm{Cl}$ atoms are displaced from the simple cubic perovskite positions by about $0.1 \AA$ and $0.2 \AA$, respectively. We further note that second order Jahn-Teller (SOJT) distortions could occur in $d^{10}$ ions as $\mathrm{Hg}^{2+}$, when the symmetry of the empty $\mathrm{n} s$ orbitals have the appropriate symmetry to mix with certain (n-1)d orbitals, but to confirm SOJT effect will require a more careful study of the pure $\mathrm{CsHgCl}_{3}$ phase.

Noting that heavily $\mathrm{Hg}$-doped $\mathrm{CsTlCl}_{3}$ has nearly simple cubic structure experimentally, we study a hypothetic cubic compound $\mathrm{Cs}_{2} \mathrm{TlHgCl}_{6}$ to investigate the effect of $\mathrm{Hg}$ doping on the electron-phonon coupling. We use a Fm-3m perovskite structure with Cs occupying (1/4 1/4 1/4), Tl $\left(\begin{array}{lll}0 & 0 & 0\end{array}\right), \mathrm{Hg}\left(\begin{array}{lll}1 / 2 & 1 / 2 & 1 / 2\end{array}\right)$ and $\mathrm{Cl}$ at $\left(\begin{array}{lll}x & 0 & 0\end{array}\right)$ and equivalent positions. Structure optimizations using HSE06 and PBE both give a value of $x \sim 0.255$ at equilibrium structure. The band structures at equilibrium structure are computed using both HSE06 and PBE and shown in Fig. 6a. Both 
HSE06 and PBE predict a metallic band structure with almost the same dispersion around the Fermi level. The separation of the $\mathrm{Tl} 6 \mathrm{~s}$ band crossing the Fermi level and the $\mathrm{Hg} 6 \mathrm{~s}$ band above the $\mathrm{Tl} 6 \mathrm{~s}$ band is about $0.5 \mathrm{eV}$ larger in the HSE06 band structure. In Fig. $6 \mathrm{~b}$ we show the PBE band structure at the equilibrium structure $x=0.255$ and at $x=0.250$ where the $\mathrm{Cl}$ atoms are displaced from the equilibrium positions. These displacements of the $\mathrm{Cl}$ atoms lead to only a small shift of the Tl $6 s$ band which crosses the Fermi level. The largest shift is along the L-W high symmetry line with only about $1.5 \mathrm{eV} / \AA$ for the reduced electron-phonon matrix element, a drastic reduction from the value of about $4.9 \mathrm{eV} / \AA$ for the parent $\mathrm{CsTlCl}_{3}$ compound. ${ }^{10}$ Since the electron-phonon coupling is proportional to the square of the reduced electron-phonon matrix element, ${ }^{2}$ therefore the $\mathrm{Hg}$ doping rapidly suppresses the electron-phonon coupling of the $\mathrm{Cl}$ breathing mode. Hence, $\mathrm{Hg}$ is a bad dopant for inducing superconductivity. The underlying reason is that $\mathrm{Hg}$ doping breaks the symmetry of the $\mathrm{Tl}-\mathrm{Cl}-\mathrm{Tl}$ bonds, leading to a different band structure around the Fermi level. In order to keep the Tl-Cl-Tl bond intact, doping the Cs site is a better choice in order to metalize the $\mathrm{CsTlCl}_{3}$ parent compound.

The metallicity of the hypothetic $\mathrm{Cs}_{2} \mathrm{TlHgCl}_{6}$ compound is however inconsistent with experimental observations that all the $\mathrm{Hg}$ doped $\mathrm{CsTlCl}_{3}$ including $\mathrm{CsHgCl}_{3}$ are insulating. It is possible that the actual structure of the doped compounds have different local distortions from what is assumed above. To investigate other possible local distortions, we construct a $2 \times 2 \times 2$ supercell of the simple cubic perovskite unit cell to study the partially doped $\mathrm{Cs}(\mathrm{Tl}, \mathrm{Hg}) \mathrm{Cl}_{3}$ materials and assume periodic condition. The supercell contains $8 \mathrm{Cs}$ atoms, $24 \mathrm{Cl}$ atoms and a total number of $8 \mathrm{Tl}$ and $\mathrm{Hg}$ atoms. We vary the number of $\mathrm{Hg}$ atoms to study different $\mathrm{Hg}$ doping levels. First of all, an odd number of $\operatorname{Hg}$ atoms $(1,3,5$, or 7$)$ results in an odd number of total electrons in the supercell, which in the band theory, results in a metallic electronic structure. This is a limitation of the relative small size of the supercell, but does not suggest that the system is metallic at these doping levels $(12.5 \%, 37.5 \%, 62.5 \%, 87.5 \%)$. Therefore, we carried out detailed studies for even number of $\mathrm{Hg}$ atoms $(2,4,6)$ which corresponds to $25 \%, 50 \%$, and $75 \%$ Hg doping. 
We start with $25 \% \mathrm{Hg}$ doping with an initial structure of $\mathrm{CsTlCl}_{3}$ in the cubic phase, i.e., the $\mathrm{Cl}$ atoms are displaced from the simple cubic perovskite positions. We have also slightly and randomly displaced all the atoms from the $\mathrm{Fm}-3 m$ cubic perovskite positions in all three directions so that the structure relaxation is not constrained to a particular space group. Since the structure relaxation can only find a local minimum, but not a global minimum, different initial configurations of the $\mathrm{Hg}$ atoms could be trapped in structures with different local minimal total energy. Therefore all the different initial configurations have to be considered. Out of the eight positions of the Tl/Hg atoms, $\left(\begin{array}{lll}0 & 0 & 0\end{array}\right),\left(\begin{array}{lll}1 / 2 & 0 & 0\end{array}\right),\left(\begin{array}{lll}0 & 1 / 2 & 0\end{array}\right),\left(\begin{array}{lll}0 & 0 & 1 / 2\end{array}\right),\left(\begin{array}{lll}1 / 2 & 1 / 2 & 0\end{array}\right),\left(\begin{array}{lll}1 / 2 & 0 & 1 / 2\end{array}\right),\left(\begin{array}{lll}0 & 1 / 2 & 1 / 2\end{array}\right),\left(\begin{array}{ll}1 / 2 & 1 / 2\end{array}\right.$ $1 / 2)$, there are only three different initial configurations for the two $\mathrm{Hg}$ atoms: they are at (1) $\left(\begin{array}{ll}0 & 0\end{array}\right.$

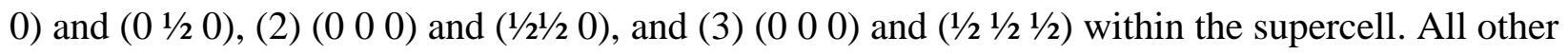
configurations are equivalent to one of these three configurations. With these initial configurations, we fully relax the atomic positions using the PBE functional. Note that both PBE and HSE06 predicted reasonable structure for $\mathrm{CsTlCl}_{3} .{ }^{12}$ Although HSE06 is slightly better than the $\mathrm{PBE}$ for the structure relaxation of $\mathrm{CsTlCl}_{3},{ }^{12}$ it is very computationally demanding. Therefore we use only PBE functional for structure relaxation in this study. After relaxation, the force acting on each atom in each direction is less than $0.1 \mathrm{meV} / \AA$. Of the three configurations after relaxation, the first one with the shortest $\mathrm{Hg}-\mathrm{Hg}$ distance has the lowest total energy, $\sim 0.2$ $\mathrm{eV} /$ supercell lower than the other two. The first one is insulating with a band gap of $0.5 \mathrm{eV}$ based on HSE06 calculations while the other two are metallic. From the viewpoint of the importance of inducing superconductivity in this phase, it is unfortunate that the metallic structures have higher total energies and are therefore not expected to be stable.

Continuing to $50 \%$ doping (4 $\mathrm{Hg}$ atoms), we consider initial configurations consisting of two pairs of $\mathrm{Hg}-\mathrm{Hg}$ atoms based on the results of $25 \%$ doping. There are three different initial configurations resulting from replacing two $\mathrm{Tl}$ atoms by two $\mathrm{Hg}$ atoms in the first configuration of the $25 \%$ doping case, i.e., the other pair of $\mathrm{Hg}-\mathrm{Hg}$ atoms are at (I) $\left(0 \frac{1}{2} 0\right)$ and $\left(1 / 21 \frac{1}{2} 0\right)$, (II) $(1 / 2$ $1 / 20)$ and $(1 / 21 / 2 \quad 1 / 2)$, and (III) $\left(\begin{array}{lll}1 / 2 & 1 / 2 & 1 / 2\end{array}\right)$ and $\left(\begin{array}{lll}0 & 1 / 2 & 1 / 2\end{array}\right)$. After relaxation, configuration (I) is 0.15 $\mathrm{eV} /$ supercell and $0.17 \mathrm{eV} /$ supercell lower in total energy than configuration (II) and configuration (III), respectively. Configuration (I) is insulating with a band gap of $\sim 1.0 \mathrm{eV}$ based on HSE06 and the other two configurations are metallic, similar to the $25 \%$ doping case. 
Now for $75 \%$ doping, there are six $\mathrm{Hg}$ atoms and two $\mathrm{Tl}$ atoms in the supercell. Here it is more convenient to consider the initial configuration of the two $\mathrm{Tl}$ atoms. Exactly the same as in the $25 \%$ doping case, the two $\mathrm{Tl}$ atoms have three different initial configurations, i.e., the two $\mathrm{Tl}$

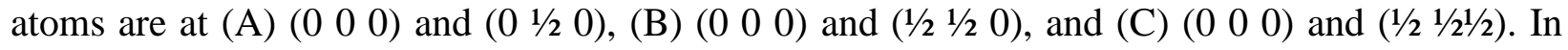
contrast to the $25 \%$ and $50 \%$ doping cases, all the three configurations have similar total energies after relaxation, differing only by $\sim 0.02 \mathrm{eV} / \mathrm{supercell}$. Moreover, all three configurations are insulators with almost the same band gap of $\sim 2.0 \mathrm{eV}$.

We further analyse the structure with the lowest total energy at each doping level. For the parent compound $\mathrm{CsTlCl}_{3}$, the $\mathrm{Cl}^{-}$ions around $\mathrm{Tl}$ show breathing distortions, resulting in alternative expanded $\mathrm{Tl}^{1+} \mathrm{Cl}_{6}$ octahedrons and compressed $\mathrm{Tl}^{3+} \mathrm{Cl}_{6}$ octahedrons. Thus the $\mathrm{Tl}^{1+3+}$ ions are in mixed valence states, whereas in $\mathrm{CsHgCl}_{3}$, the $\mathrm{Hg}^{2+}$ ions are in a single valence state. A common theme of the structures of the doped $\mathrm{Cs}(\mathrm{Tl}, \mathrm{Hg}) \mathrm{Cl}_{3}$ compounds found in the supercell calculations is that, there are equal amount of $\mathrm{Tl}^{3+}$ and $\mathrm{Tl}^{1+}$ ions with breathing-distorted $\mathrm{Cl}^{-}$ions surrounding them, while the $\mathrm{Cl}^{-}$ions around the $\mathrm{Hg}^{2+}$ ions form SOJT distorted octahedrons, similar to the Jahn-Teller distortion in the parent compound $\mathrm{CsAuCl}_{3}$. In the $50 \%$ doping case, the material shows a quasi-layered structure with $\mathrm{Hg}$ atoms in the $z=0$ plane and $\mathrm{Tl}$ atoms in the $z=1 / 2$ plane. This structure could be difficult to form in practice. Experimentally, 50\% $\mathrm{Hg}$-doped sample was not made successfully.

The evolution of the total density of states from $\mathrm{CsTlCl}_{3}$ to $\mathrm{CsHgCl}_{3}$ is shown in Fig. 7. With increasing the concentration of $\mathrm{Hg}$, the main peak below the Fermi level, which consists mainly on $\mathrm{Cl} \mathrm{3p} \mathrm{states,} \mathrm{moves} \mathrm{towards} \mathrm{the} \mathrm{Fermi} \mathrm{level,} \mathrm{consistent} \mathrm{with} \mathrm{removing} \mathrm{electrons} \mathrm{from} \mathrm{the}$ materials ( $\mathrm{Hg}$ has one less electron than $\mathrm{Tl}$ ). The band gap, computed by DFT-HSE06, first decreases from $\sim 1.2 \mathrm{eV}$ in $\mathrm{CsTlCl}_{3}$ to $\sim 0.5 \mathrm{eV}$ in the $25 \% \mathrm{Hg}$ doped compound, and increases to $\sim 1.0 \mathrm{eV}$ and $\sim 2.0 \mathrm{eV}$ in $50 \%$ and $75 \%$ doped compounds respectively, and finally decreases again to $\sim 0.6 \mathrm{eV}$ in $\mathrm{CsHgCl}_{3}$. Small doping of $\mathrm{Hg}$ into $\mathrm{CsTlCl}_{3}$ weakens the $\mathrm{Cl}$ breathing distortion centred at $\mathrm{Tl}$ atoms which reduces the mixed-valence of $\mathrm{Tl}$ atoms. As a result, the band gap decreases with small $\mathrm{Hg}$ doping, as shown in Fig. 7. On the other hand, small $\mathrm{Tl}$ doping into 
$\mathrm{CsHgCl}_{3}$ introduces large distortions of the $\mathrm{Cl}$ octahedra centred both at $\mathrm{Hg}$ and $\mathrm{Tl}$ atoms. The large distortions induced by $\mathrm{Tl}$ doping act to increase the band gap rapidly, as shown in Fig. 7 where $25 \% \mathrm{Tl}$ doping increases the band gap from $\sim 0.6 \mathrm{eV}$ in $\mathrm{CsHgCl}_{3}$ to $\sim 2.0 \mathrm{eV}$. Based on our study, $\mathrm{Hg}$ doping into $\mathrm{CsTlCl}_{3}$ is unable to suppress the Cl-breathing distortions and mixedvalence of $\mathrm{Tl}$, hence it is unable to metallize the parent compound.

\section{Conclusions}

We have prepared the solid solution $\mathrm{CsTl}_{1-\mathrm{x}} \mathrm{Hg}_{\mathrm{x}} \mathrm{Cl}_{3} . \mathrm{x}=0.1$ is tetragonal, $\mathrm{x}=0.2$ is cubic with Fm$3 \mathrm{~m}$ space group and two crystallographic positions for $\mathrm{Tl}^{1+}$ and $\mathrm{Tl}^{3+}, \mathrm{x}=0.4$ and 0.5 cannot be prepared as single phases, $\mathrm{x}=0.6$ and 0.8 are simple cubic perovskites, $\mathrm{Pm}-3 \mathrm{~m}$ and one single position for $\mathrm{Tl}^{1+}, \mathrm{Tl}^{3+}$ and $\mathrm{Hg}^{2+}$. All the samples are insulating and there is no sign of superconductivity. By X-ray absorption spectroscopy we observe an intermediate $\mathrm{Tl}$ valence state for all the series, confirming the mixed valence of $\mathrm{Tl}^{1+}$ and $\mathrm{Tl}^{3+}$. Raman Spectroscopy shows the presence of active Tl-Cl-Tl stretching mode and the appearance of $\mathrm{Tl}-\mathrm{Cl}-\mathrm{Hg}$ mode when the content of $\mathrm{Hg}$ increases. Theoretical calculations confirm that the ground states of all $\mathrm{CsTl}_{1-\mathrm{x}} \mathrm{Hg}_{\mathrm{x}} \mathrm{Cl}_{3}$ phases are insulating and $\mathrm{Hg}$ doping cannot produce superconductivity. 
Table 1: Crystal data and atomic parameters for $\mathrm{CsTl}_{1-x} \mathrm{Hg}_{x} \mathrm{Cl}_{3}$ at $100 \mathrm{~K}$ for $x=0.2,0.6$ and 0.8 . The anisotropic displacement factor exponent takes the form: $-2 \pi^{2}\left[h^{2} a^{* 2} U_{11}+\ldots+2 h k a^{*} b^{*} U_{12}\right]$, with $U_{i j}$ in $\AA^{2}$.

\begin{tabular}{|c|c|c|c|c|}
\hline & $x=0.2$ & & $x=0.6$ & $x=0.8$ \\
\hline $\begin{array}{l}\mathbf{F m}-\mathbf{3 m} \\
a / \AA \\
V / \AA^{3}\end{array}$ & $\begin{array}{l}10.804(2) \\
1261.3(8)\end{array}$ & $\begin{array}{l}\mathbf{P m - 3 m} \\
\mathrm{a} / \AA^{\circ} \\
\mathrm{V} / \AA^{3}\end{array}$ & $\begin{array}{l}5.3739(14) \\
155.19(12)\end{array}$ & $\begin{array}{l}5.3750(11) \\
155.29(6)\end{array}$ \\
\hline $\begin{array}{l}\operatorname{Cs}(\mathbf{1}) \mathbf{8 c}\left(1 / \mathbf{1}^{1 / 4} \mathbf{1} / \mathbf{4}\right) \\
U_{11} / \AA^{2} \\
\text { Occupancy }\end{array}$ & $\begin{array}{l}0.0855(16) \\
1.000 .\end{array}$ & $\begin{array}{l}\text { Cs } 1 \mathbf{1} a(\mathbf{0} 00) \\
U_{11} \AA^{2} \\
\text { Occupancy }\end{array}$ & $\begin{array}{l}0.0472(9) \\
1.000\end{array}$ & $\begin{array}{l}0.0371(41) \\
1.000\end{array}$ \\
\hline $\begin{array}{l}\mathbf{T l} / \mathbf{H g}(\mathbf{1}) \mathbf{4} \boldsymbol{a}(\mathbf{0} 0 \mathbf{0}) \\
U_{11} / \AA^{2} \\
\text { Occupancy }\end{array}$ & $\begin{array}{l}0.0286(6) \\
1.000\end{array}$ & $\begin{array}{l}\mathbf{T l} / \mathbf{H g} \mathbf{H}(\mathbf{A}) \mathbf{b} \boldsymbol{b}\left(1 / 2 \mathbf{2}^{1 / 2} \mathbf{2} / 2\right) \\
U_{11} / \AA^{2} \\
\text { Occupancy }\end{array}$ & $\begin{array}{l}0.0216(5) \\
0.880(8)\end{array}$ & $\begin{array}{l}0.0160(3) \\
0.947(5)\end{array}$ \\
\hline $\begin{array}{l}\mathbf{H g}(\mathbf{2 A}) \mathbf{4 b}(1 / 2 \mathbf{0} 0) \\
U_{11} / \AA^{2} \\
\text { Occupancy } \\
\mathbf{T l}(\mathbf{2 B}) \mathbf{2 4 b}(\mathbf{x} 00) \\
x \\
U_{\text {iso }} / \AA^{2} \\
\text { Occupancy }\end{array}$ & $\begin{array}{l}0.0028(16) \\
0.167\end{array}$ & $\begin{array}{l}\mathbf{T} \mathbf{l} / \mathbf{H g}(\mathbf{1 B}) \mathbf{6} f\left(\mathbf{x}^{1 / 2} \mathbf{1} 1 / 2\right) \\
x \\
U_{11} / \AA^{2} \\
\text { Occupancy }\end{array}$ & $\begin{array}{l}0.346(5) \\
0.0216(5) \\
0.120(8)\end{array}$ & $\begin{array}{l}0.352(7) \\
0.0160(3) \\
0.053(5)\end{array}$ \\
\hline $\begin{array}{l}\mathbf{C l}(\mathbf{1} \mathbf{A}) \mathbf{4 e}(\mathbf{x} \mathbf{0} 0) \\
x \\
U_{11} / \AA^{2} \\
U_{12} / \AA^{2} \\
\text { Occupancy } \\
\mathbf{C l}(\mathbf{1 B}) \mathbf{4 8 h}(\mathbf{y} \mathbf{y} \mathbf{0}) \\
y \\
U_{11} / \AA^{2} \\
U_{12} / \AA^{2} \\
\text { Occupancy }\end{array}$ & $\begin{array}{l}0.2413(9) \\
0.056(5) \\
0.059(3) \\
0.833\end{array}$ & $\begin{array}{l}\text { Cl 3c( (0 1/2 1/2) } \\
U_{11} / \AA^{2} \\
U_{12} / \AA^{2} \\
\text { Occupancy }\end{array}$ & $\begin{array}{l}0.088(7) \\
0.065(4) \\
1.000\end{array}$ & $\begin{array}{l}0.099(5) \\
0.069(2) \\
1.000\end{array}$ \\
\hline $\begin{array}{l}\text { R1 } \\
\text { wR2 }\end{array}$ & $\begin{array}{l}0.0513 \\
0.1142\end{array}$ & $\begin{array}{l}\text { R1 } \\
\text { wR2 }\end{array}$ & $\begin{array}{l}0.0337 \\
0.0663\end{array}$ & $\begin{array}{l}0.0246 \\
0.0637\end{array}$ \\
\hline
\end{tabular}


Table 2: Main interatomic distances $(\AA)$ for $\mathrm{CsTl}_{1-\mathrm{x}} \mathrm{Hg}_{\mathrm{x}} \mathrm{Cl}_{3}$ series at $100 \mathrm{~K}$.

\begin{tabular}{|ll|lll|}
\hline $\mathrm{F} m-3 m$ & $x=0.2$ & $\mathrm{P} m-3 m$ & $x=0.6$ & $x=0.8$ \\
\hline $\mathrm{Cs}(1 \mathrm{~A})-\mathrm{Cl}(1 \mathrm{~A}) \times 12$ & $3.821(1)$ & $\mathrm{Cs}-\mathrm{Cl} \times 12$ & $3.800(1)$ & $3.801(1)$ \\
$\mathrm{Cs}(1 \mathrm{~A})-\mathrm{Cl}(1 \mathrm{~B}) \times 6$ & $3.01(3)$ & & & \\
& & & & \\
$\mathrm{Tl} / \mathrm{Hg}(1)-\mathrm{Cl}(1 \mathrm{~A}) \times 6$ & $2.61(1)$ & $\mathrm{Tl} / \mathrm{Hg}(1 \mathrm{~A})-\mathrm{Cl} \times 6$ & $2.687(1)$ & $2.688(1)$ \\
$\mathrm{Tl} / \mathrm{Hg}(1)-\mathrm{Cl}(1 \mathrm{~B}) \times 6$ & $2.50(7)$ & $\mathrm{Tl} / \mathrm{Hg}(1 \mathrm{~B})-\mathrm{Cl} \times 4$ & $2.812(9)$ & $2.802(11)$ \\
& & $\mathrm{Tl} / \mathrm{Hg}(1 \mathrm{~B})-\mathrm{Cl} \times 1$ & $3.515(9)$ & $3.483(11)$ \\
$\mathrm{Tl} / \mathrm{Hg}(2 \mathrm{~A})-\mathrm{Cl}(1 \mathrm{~A}) \times 6$ & $2.79(1)$ & & & \\
$\mathrm{Tl} / \mathrm{Hg}(2 \mathrm{~B})-\mathrm{Cl}(1 \mathrm{~A}) \times 4$ & $2.90(1)$ & & & \\
\hline
\end{tabular}




\section{Acknowledgment}

This work was supported by the NSF-DMR-0966829 grant. Z.P.Y and G.K. were supported by the AFOSR-MURI program towards better and higher temperature superconductors. We gratefully acknowledge the high resolution X-ray powder diffraction data collected at the beam

line X16C, N.S.L.S., Brookhaven National Laboratory, supported by the US DOE, Office of Science, Office of Basic Energy Sciences, under contract No. DE-AC02-98CH10886. The work at IOPCAS was supported by NSF \& MOST research grants. 
*martha@ rutchem.rutgers.edu

\# Current address: Nanoscience Center and Science Center, Niels Bohr Institute, University of Copenhagen, Denmark

$\S$ Current address: Uppsala University, Department of Engineering Sciences, Division of Solid State Physics, Uppsala, Sweden

Supporting Information Available: Includes the crystallographic data and structure refinement of the samples using Single Crystal x-ray Diffraction. This material is available free of charge via the Internet at http://pubs.acs.org." 


\section{References}

(1) Cava, R.; Batlogg, B.; Krajewski, J.; Farrow, R.; Rupp, L.; White, A.; Short, K.; Peck W.; Kometani, T. Nature, 1988, 332, 814-816.

(2) Yin, Z. P.; Kutepov, A.; Kotliar, G. Phys. Rev. X 2013, 3, 021011.

(3) Baumert, B. J. Supercond. 1995, 8, 175-181.

(4) Pei, S.; Jorgensen, J. D.; Dabrowski, B.; Hinks, D. G.; Richards, D. R.; Mitchell, A.W.; Newsam, J. M.; Sinha, S.

K.; Vaknin D.; Jacobson, A. J. Phys. Rev. B 1990, 41, 4126-4141.

(5) Climent-Pascual, E.; Ni, N.; Jia, S.; Huang Q.; Cava, R. Phys. Rev. B 2011, 83, 174512.

(6) N.Kojima,; Hasegawa, M.; Kitagawa, H.; Kikegawa, T.; Shimomura, O. J. Am. Chem. Soc. 1994, 116, 25, 11368-11374.

(7) Kitagawa, H.; Sato, H.; Kojima, N.; Kikegawa, T.; Shimomura, O. Solid State Commun. 1991, 78, 989-995.

(8) Kojima N.; Kitagawa, H. J. Chem. Soc. Dalton Trans. 1994, 3, 327-331.

(9) Wang, S.; Hirai, S.; Shapiro, M. C.; Riggs, S. C.; Geballe, T. H.; Mao, W. L.; Fisher, I. R. Phys. Rev. B 2013, 87, 054104.

(10) Yin, Z. P.; Kotliar, G. EPL 2013, 101, 27002.

(11) Schoop, L. M.; Müchler, L.; Felser, C.; Cava, R. J. Inorg. Chem. 2013, 52 (9), 5479-5483

(12) Retuerto, M.; Emge, T.; Li, M. R.; Yin, Z. P.; Croft, M.; Ignatov, A.; Simonson, J.; Aronson, M.; Stephens, P.;

Hadermann, J.; Pan, A.; Basov, D. N.; Kotliar, G.; Greenblatt, M. Chem. Mater. 2013, 25(20), 4071-4079.

(13) Khan, Y.; Nahm, K.; Rosenberg, M.; Willner, H. Phys. Status Solidi A 1977, 39, 79-88.

(14) Coelho Software. TOPAS-Academic V5, Brisbane, Australia 2012.

(15) Bruker. SADABS, Bruker-AXS Inc., Madison, Wisconsin, USA 2013.

(16) Sheldrick, G. M. Acta Cryst. A 2008, 64, 112-122.

(17) Petricek, V.; Dusek, M.; Palatinus, L. Jana 2006. Institute of Physics, Prague, Czech Republic.

(18) Huan, G.; Greaney, M.; Greenblatt, M.; Liang G.; Croft, M. Solid State Ionics 1988, 32-33,134-140.

(19) Li, S.; Greenblatt, M.; Jeon, Y.; Chen, J.; Liang G.; Croft, M. Physica C 1991, 173, 239-244.

(20) Matsushita, N.; Ahsbahs, H.; Hafner, S.S.; Kojima, N, J. Solid State Chem. 2007, 180, 1353-1360. 
(21) Bill, J.; Lerch, K.; Laqua, W. Z. Anorg. Allg. Chem. 1990, 589, 7-11.

(22) Ferrari, A. Gazzetta Chimica Italiana 1937, 67, 94-98.

(23) Shannon, R.D. Acta Cryst. A 1976, 32, 751-767.

(24) Li S.; Greenblatt, M.; Jeon, Y.; Chen, J.; Croft, M. Physica C 1991, 173, 239-244.

(25) Yang, T.; Abakumov, A.; Hadermann, J.; Van Tendeloo, G.; Nowik, I.; Stephens, P.; Hemberger, J.; Tsirlin, A.;

Ramanujachary, K.; Lofland, S.; Croft, M.; Ignatov, A.; Sun, J.; Greenblatt, M. Chem. Sci. 2010, 1, 751-762.

(26) Heald, S. M.; DiMarzio, D.; Croft, M.; Hegde, M. S.; Li, S.; Greenblatt, M. Phys. Rev. B 1989, 40, 8828-8833.

(27) Glaser, T.; Hedman, B.; Hodgson, K.; Solomon, E. I. Acc. Chem. Res. 2000, 33, 859-868.

(28) Kozimor, S. A.; Yang, P.; Batista, E. R.; Boland, K. S.; Burns, C. J.; Clark, D. L.; Conradson, S. D.; Martin, R.

L.; Wilkerson, M. P.; Wolfsberg, L. E. J. Am. Chem. Soc. 2009, 131, 12125-12136

(29) Pakhomov, V.I.; Goryunov, A.V. Zhurnal Neorganicheskoi Khimii 1993, 38, 1501-1508

(30) Perdew, J. P.; Burke, K.; Ernzerhof, M. Phys. Rev. Lett. 1996, 77, 3865-3868.

(31) Krukau, A. V.; Vydrov, O. A.; Izmaylov, A. F.; Scuseria, G. E. J. Chem. Phys. 2006, 125, 224106.

(32) Kresse G.; Furthmuller, J. Comput. Mat. Sci. 1996, 6, 15-50. 


\section{Figure Captions}

Fig. 1: PXD patterns of $\mathrm{CsTl}_{1-\mathrm{x}} \mathrm{Hg}_{\mathrm{x}} \mathrm{Cl}_{3}$. Upper inset shows the crystals prepared. Lower inset shows a zoom of the low angle area to highlight the superstructure peaks.

Fig. 2: SPXD patterns of $\mathrm{CsTl}_{1-\mathrm{x}} \mathrm{Hg}_{\mathrm{x}} \mathrm{Cl}_{3}$ for $x=0.1,0.2$, and 0.4 . The black trace is raw data, red is Rietveld refinement, and blue is the difference (linear scale at right). In the top panel, the dense row of tick marks are allowed peak positions of the tetragonal phase, the middle row is the cubic phase, and the bottom row is the diamond powder used for dilutant and internal standard. Green arrows in the $x=0.2$ and $x=0.6$ panels indicate the (111) peak present in the doubled Fm$3 m$ phase which would be absent for the $P m-3 m$ primitive cell.

Fig.3: (a) Tl- $\mathrm{L}_{3}$ edges of $\mathrm{CsTlCl}_{3}, \mathrm{CsTl}_{0.2} \mathrm{Hg}_{0.8} \mathrm{Cl}_{3}, \mathrm{Tl}^{3+}$ standards $\left(\mathrm{Tl}_{2} \mathrm{O}_{3}\right.$ and $\left.\mathrm{Cs}_{2} \mathrm{TlCl}_{5}\right)$ and $\mathrm{Tl}^{1+}$ standard $\mathrm{TlCl}$. Inset: expanded view of $\mathrm{Tl}^{-\mathrm{L}_{3}}$ pre-edge region with a feature related to empty $6 \mathrm{~s}-$ final states, background was subtracted. (b) $\mathrm{Cl}-\mathrm{K}$ edges of $\mathrm{CsTlCl}_{3}-\mathrm{t}, \mathrm{CsTlCl}_{3}-\mathrm{c}, \mathrm{CsHgCl}_{3}$ and standards. Note $\mathrm{KClO}_{3}$ spectrum has been multiplied by a factor of 0.8 to facilitate comparison on a similar vertical scale. The prominent, pre-edge, s-hole related features of all compounds (except $\mathrm{s}^{2}$ of $\mathrm{TlCl}$ ) are noted. (c) Comparison of $\mathrm{Cl}-\mathrm{K}$ pre-edges of $\mathrm{CsTl}_{1-\mathrm{x}} \mathrm{Hg}_{\mathrm{x}} \mathrm{Cl}_{3}$ compounds. Note the systematic transfer of spectral weight between A-feature and B-feature with increasing Hg content.

Fig. 4: Evolution of the Raman Spectra of $\mathrm{CsTl}_{1-x} \mathrm{Hg}_{x} \mathrm{Cl}_{3}$

Fig. 5: Band structure of (a) face-centered cubic $\mathrm{CsTlCl}_{3}$ and (b) simple cubic $\mathrm{CsHgCl}_{3}$ using DFT-PBE and DFT-HSE06

Fig. 6: Band structure of the hypothetic face-centered cubic $\mathrm{Cs}_{2} \mathrm{TlHgCl}_{6}$ using DFT-PBE and/or DFT-HSE06 at (a) equilibrium structure $(\mathrm{x}=0.255)$ and (b) at $\mathrm{x}=0.255$ and $\mathrm{x}=0.250$. 
Fig. 7: Total density of states (DOS) per five atoms for $\mathrm{CsTlCl}_{3}, \mathrm{CsTl}_{0.75} \mathrm{Hg}_{0.25} \mathrm{Cl}_{3}$, $\mathrm{CsTl}_{0.5} \mathrm{Hg}_{0.5} \mathrm{Cl}_{3}, \mathrm{CsTl}_{0.25} \mathrm{Hg}_{0.75} \mathrm{Cl}_{3}$, and $\mathrm{CsHgCl}_{3}$ computed by DFT-PBE and DFT-HSE06. 
Figures

Fig. 1

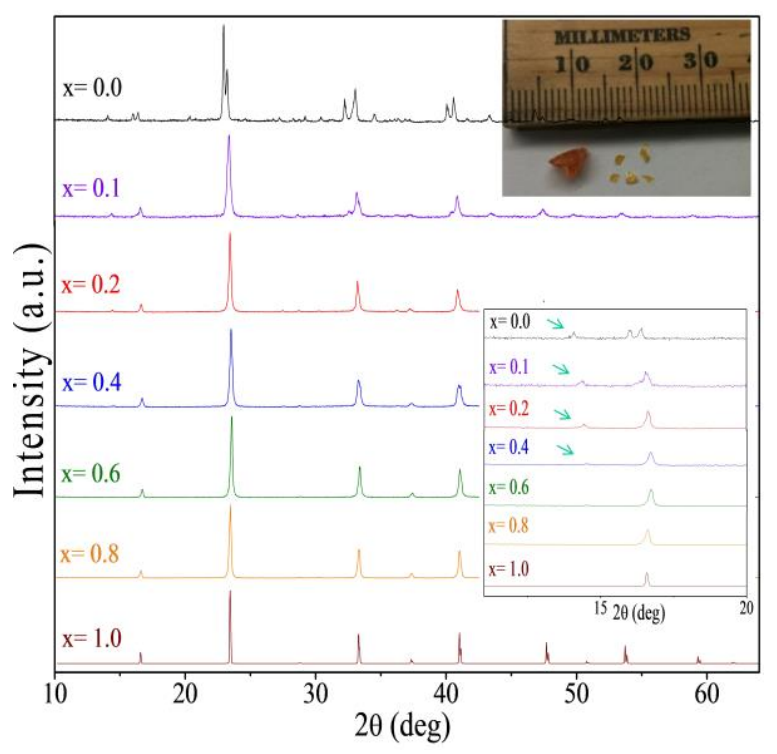


Fig. 2

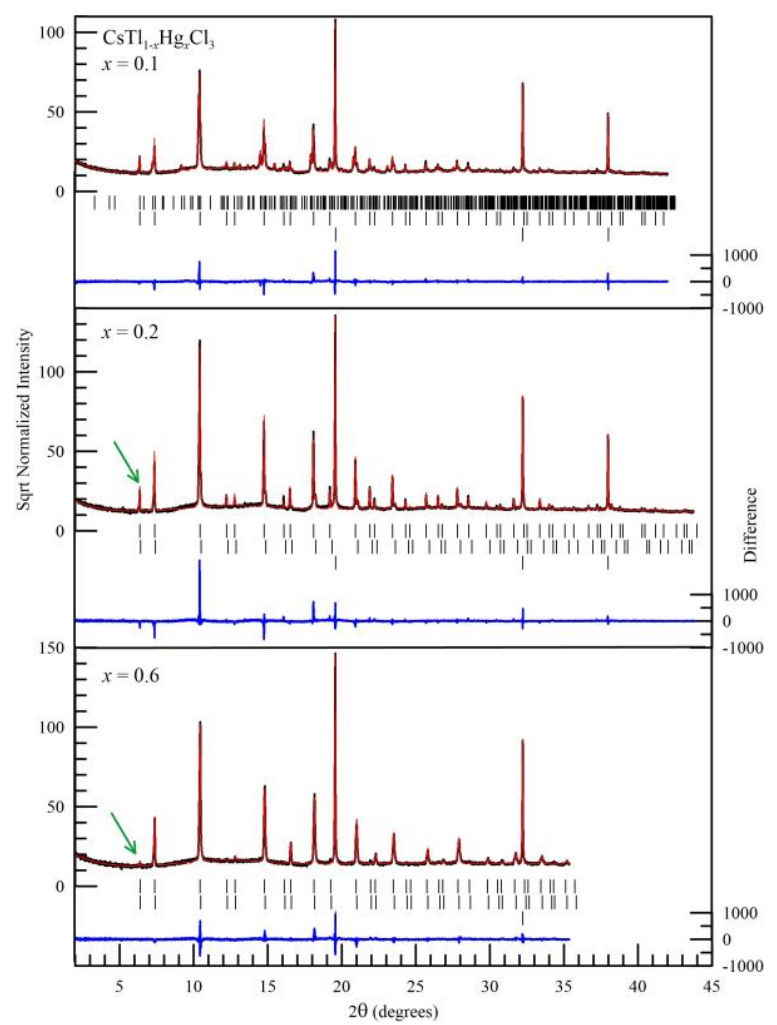


Fig. 3
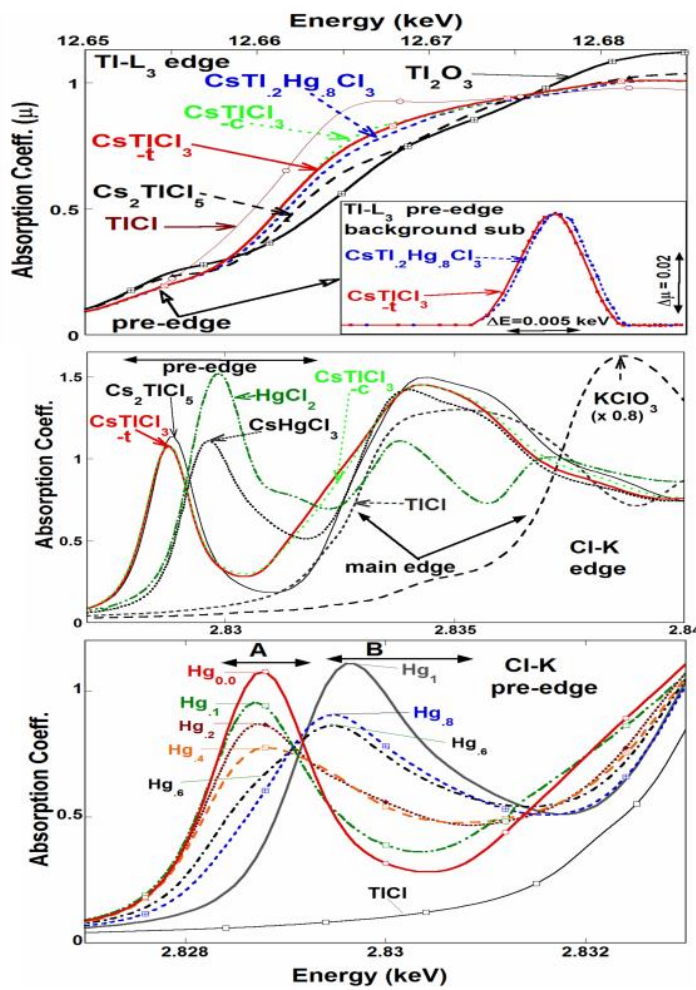
Fig. 4

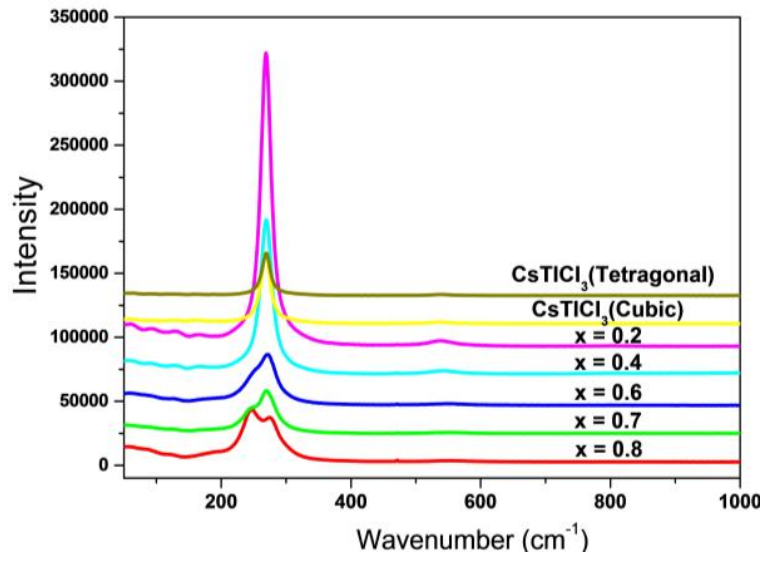


Fig. 5
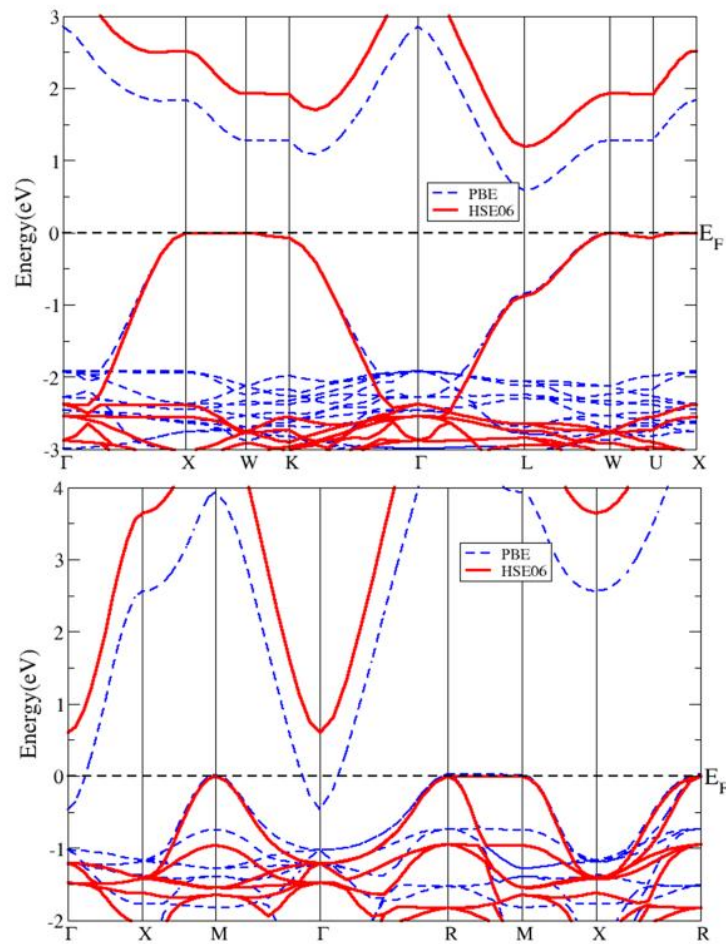
Fig. 6
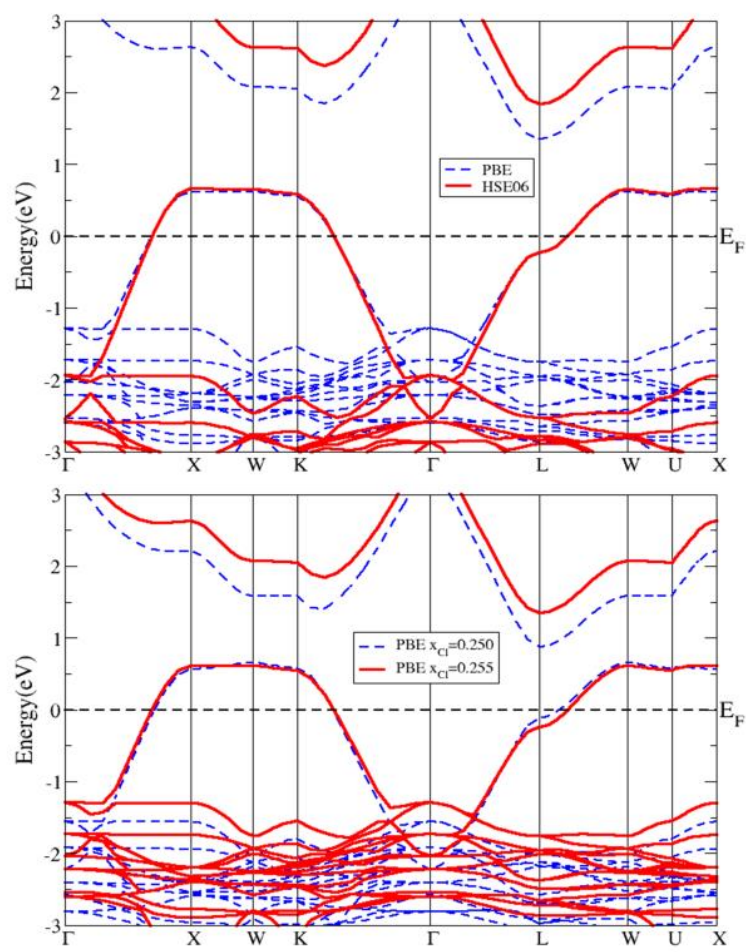
Fig. 7

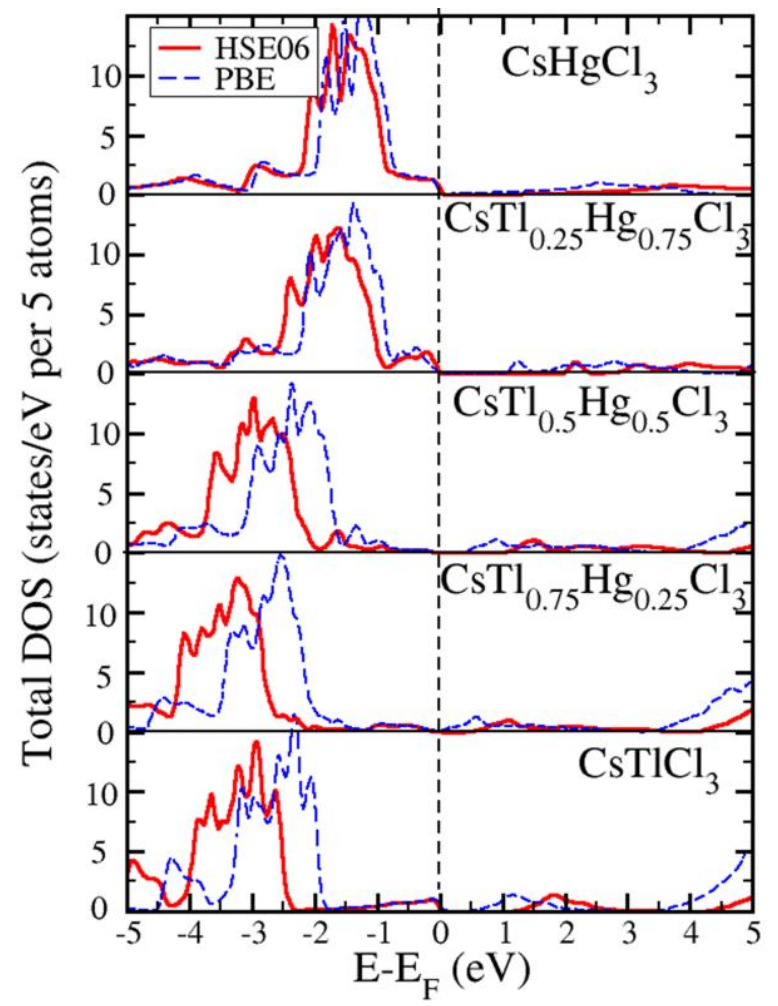




\section{Table of Contents synopsis}
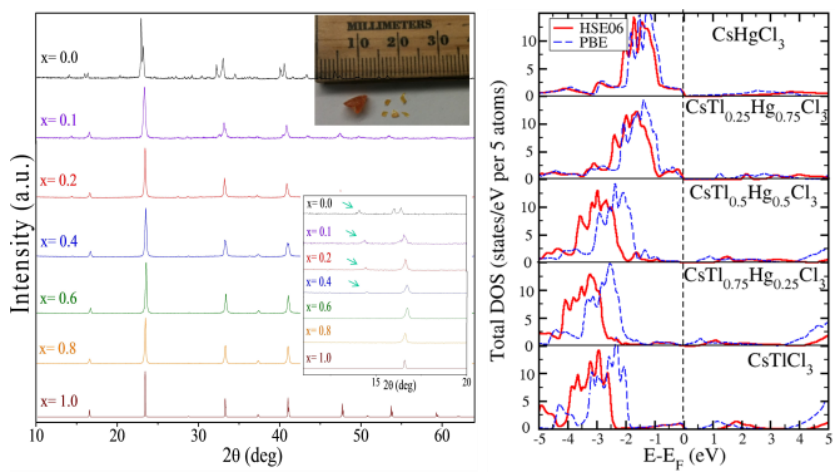

$\mathrm{CsTl}_{1-x} \mathrm{Hg}_{x} \mathrm{Cl}_{3}(x=0.0,0.1,0.2,0.4,0.6$ and 0.8$)$ perovskites were prepared via substitution of $\mathrm{Tl}$ by $\mathrm{Hg}$ for possible superconductivity. Two phase transitions are observed with $x$ evolution: from tetragonal $I 4 / m$ as in $\mathrm{CsTlCl}_{3}$ into a cubic $F m-3 m$ structure when $x \geq 0.2$, and finally primitive cubic $P m-3 m$ for $x \geq 0.6$. No solid solution forms for $x=0.4$ and 0.5 . The mixed valence state of $\mathrm{Tl}^{1+}$ and $\mathrm{Tl}^{3+}$ is confirmed by the x-ray absorption spectroscopy. Raman spectroscopy shows the presence of the Tl-Cl-Tl stretching mode over the whole series and the intensity of the $\mathrm{Tl}-\mathrm{Cl}-\mathrm{Hg}$ mode increases with increasing $\mathrm{Hg}$ content. First principle calculations confirmed that the phases are insulators in their ground state, and $\mathrm{Hg}$ is not a good dopant in the search of superconductivity in this system. 